\title{
A Geant4-based Monte Carlo Tool for Nuclear Astrophysics
}

D. Lattuada ${ }^{1, \star}$, M. La Cognata ${ }^{2}$, A. Anzalone ${ }^{2}$, D. L. Balabanski ${ }^{1}$, S. Chesnevskaya ${ }^{1}$, M. Costa $^{2}$, V. Crucillà ${ }^{2}$, G.L. Guardo ${ }^{1}$, M. Gulino ${ }^{2,3}$, C. Matei ${ }^{1}$, R.G. Pizzone ${ }^{2}$, S. Romano ${ }^{2,4}$, C. Spitaleri ${ }^{2}, A$. Tumino ${ }^{2,3}$, and $Y . \mathrm{Xu}^{1}$.

${ }^{1}$ ELI-NP / IFIN-HH, Magurele, Romania.

${ }^{2}$ INFN, Laboratori Nazionali del Sud, Catania, Italy.

${ }^{3}$ Facoltà di Ingegneria e Architettura, Università Kore, Enna, Italy.

${ }^{4}$ Dipartimento di Fisica e Astronomia, Università degli Studi, Catania, Italy.

\begin{abstract}
Present and future gamma-beam facilities represent a great opportunity to validate and evaluate the cross-sections of many photonuclear reactions at near-threshold energies, whose data mostly come from theoretical calculations. We developed a Monte Carlo (MC) software that makes use of the validated tracking Geant4 libraries and the n-body event generator of ROOT libraries in order to provide a fast, realiable and complete MC tool to be used for nuclear physics experiments, with a particular focus on photo-nuclear processes. We discuss the results of the MC simulations performed in order to evaluate the effects of the electromagnetic background, the straggling of the emitted particles due to the target thickness and the resolution of the silicon detectors. Finally we present the preliminary results on some nuclear reactions involved in the p-process, which will be studied with ELISSA and the GBS at ELI-NP.
\end{abstract}

\section{Introduction}

Monte Carlo (MC) simulations have always played a crucial role in preparation of experiments, in data analysis and for proper understanding of the experimental results. With the development of new experimental methods and techniques, as well as more sensitive and complex detectors, we experience a growing need for details in the simulations. In this scenario the lack of a complete, validated, fast and open-source software tool to be jointly (commonly) used in nuclear physics experiments reflects the complexity of comparing data coming from different experiments. Therefore we are developing such a tool, using open-source scientific libraries with a strong record of validation in various fields of physics. This tool is intended to be used for studying photonuclear reactions at $\gamma$-beam facilities with ELISSA (Extreme Light Infrastructre Silicon Strip Array [1, 2]), a new silicon-strip detector array under development at the Extreme Light Infrastructure - Nuclear Physics (ELI-NP [3]) laboratory, but any detector and any nuclear reaction can be used. The idea of this toolkit came from the current lack of validated photonuclear physics working at low energies in Geant4 and the need of a Geant4-based code to provide detailed information on electromagnetic (and hadronic)

^e-mail: dario.lattuada@eli-np.ro 


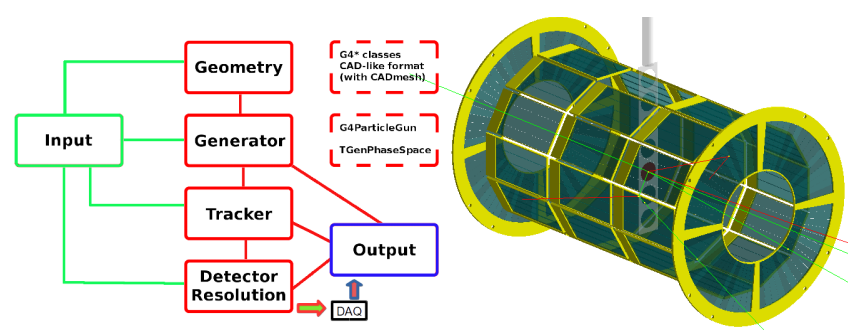

Figure 1. Left: A scheme of the MC code. Right: The geometry of ELISSA imported in GROOT. Gamma (green) and electron (red) tracks are shown.

background in nuclear physics experiments. The open-source GROOT [4] (Geant4 [5] and ROOT [6] Object-Oriented Toolkit) code we are currenlty developing mainly relies on ROOT, Geant4 and the Standard Template Library (STL) of $\mathrm{C}++$.

\section{Results}

The tool has been and will be used for past and future experiments. With ELISSA at ELI-NP, it will be possible to study photonuclear reactions of great importance for astrophyisics. Here, we present some preliminary results obtained with GROOT involving the p-process, i.e. the mechanism thought to be responsible for the abundances of the so-called p-nuclei, stable proton-rich nuclides far from the stability line, typically less abundant by factors of ten to one thousand than the other isotopes of the same element. The used photonuclear reaction rates of $(\gamma, \alpha)$ and $(\gamma, p)$ reactions are computed in [7] with the help of TALYS[8]. The choice of the model is to be considered as an example, since the generated events are weighted a posteriori. In this way, it is very easy to compare different model predictions for the same reaction (and we did it using other approaches in the past [4]), by simply renormalizing the reaction rates according to the evaluated cross-sections. We examine the ${ }^{92} \mathrm{Mo}(\gamma, \alpha)^{88} \mathrm{Zr}$ and ${ }^{92} \mathrm{Mo}(\gamma, p)^{91} \mathrm{Nb}$ photonuclear reactions at $9 \mathrm{MeV}$, of great interest for the p-process. The latter is very difficult to study in the inverse $(p, \gamma)$ reaction, because of the very limited availability of ${ }^{91} \mathrm{Nb}$ targets and beams.

In figure 2, the recorded energy of the ejected particles is plotted (ELISSA setup is shown in figure 1 for reference, only the X3 "rings" are considered). The data are plotted after applying a Gaussian smearing with an energy-dependent sigma in order to simulate the detector energy resolution as reported from Micron and calculated in $[9,10]$. Despite the straggling in the target, the expected alpha-signal is well separated from the proton one, which in turn is (calculated to be) much greater, by nearly 9 orders of magnitude. Theta vs. alpha spectra are used to disentangle the proton and alpha signals owing to the different kinematics, similarly to how we did in [4]. The electromagnetic background is calculated for a setup with a 25 $\mu \mathrm{m}$ thick aluminum flange foil placed $3 \mathrm{~m}$ far from a thin $3 \mu \mathrm{m}{ }^{92}$ Mo target on a $0.5 \mu \mathrm{m}$ carbon backing (black squares on figure 2, improvements on statistics are ongoing). By using an additional turbopump we can have 3 meters of beam line and the chamber under the same vacuum conditions, so that the photons will only encounter one thin window between the $\gamma$ transport line and the scattering chamber at pressures of $10^{-3}$ mbar e $10^{-6}$ mbar respectively.

In conclusion, the validation of the tool is still in progress and we are currently analizing our experimental results from various tests and experiments, in order to compare them with 


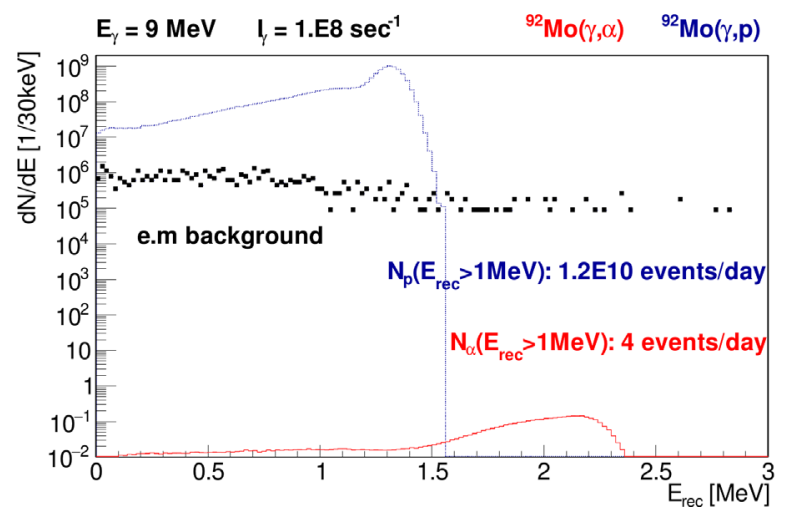

Figure 2. Alphas (red line) and protons (blue dotted line) coming from the $\gamma+{ }^{92}$ Mo reaction at 9 $\mathrm{MeV}$ beam with ELI-NP GBS specification. The black squares represent the evaluated e.m. background. $\mathrm{I}_{\gamma}$ is a conservatory estimate of the beam intensity, the number of generated background events thus roughly corresponds to 1 second livetime. further simulations of the e.m. background are going in order to increase the statistics

the simulations. Data from experiments are crucial in order to validate our tool and we look forward for future collaborations with international groups. Nevertheless, the reasonable results we show here are realiable since they are obtained using widely validated libraries and classes from Geant4 and ROOT. The code is currently under development at ELI-NP and due to its high level of customizability and ease to use it is applying to become the reference MC tool for photonuclear experiments with silicon detectors at this facility.

We acknowledge the support from the Extreme Light Infrastructure Nuclear Physics (ELI-NP) Phase II, a project cofinanced by the Romanian Government and the European Union through the European Regional Development Fund - the Competitiveness Operational Programme (1/07.07.2016, COP, ID 1334).

\section{References}

[1] O. Tesileanu et al., 2016 Charged particle detection at ELI-NP, Rom. Rep. Phys. 68 S699

[2] M. La Cognata et al., 2017 Journal of Instrumentation 12 C03079

[3] D. Filipescu et al., Eur. Phys. J., A51, 185 (2015)

[4] D. Lattuada et al., EPJ Web of Conferences 165, 01034 (2017)

[5] Geant4: A Simulation toolkit - Geant4 Collaboration (Agostinelli, S. et al.), Nucl. Inst. \& Meth. A506 (2003) 250-303 SLAC-PUB-9350, FERMILAB-PUB-03-339.

[6] ROOT - An Object Oriented Data Analysis Framework - Rene Brun and Fons Rademakers, Proceedings AIHENP'96 Workshop, Lausanne, Sep. 1996, NIM-A 389 (1997) 81-86.

[7] Y. Xu et al., EPJ Web of Conferences 146, 01015 (2017)

[8] A.J. Koning, S. Hilaire, M.C. Duijvestijn, "TALYS-1.0", in Proceedings of the International Conference on Nuclear Data Science Technology 2007, EDP Sciences Nice, France (2008), p. 211-214.

[9] G. L. Guardo et al., EPJ Web of Conferences 165, 01026 (2017)

[10] S. Chesnevskaya et al., EPJ Web of Conferences 165, 01011 (2017) 